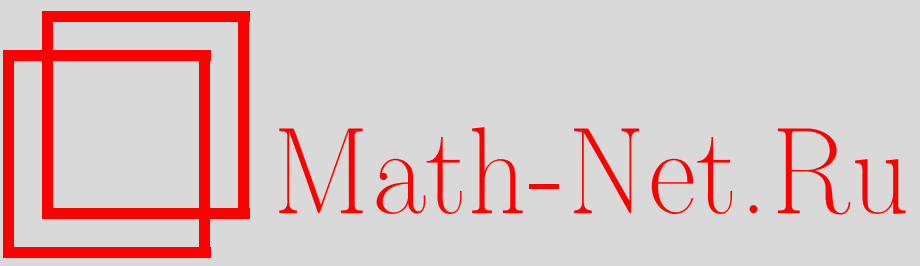

О. В. Маркова, О длине алгебры верхнетреугольных матриц, УМН, 2005, том 60, выпуск 5, 177-178

DOI: https://doi.org/10.4213/rm1653

Использование Общероссийского математического портала Math-Net.Ru подразумевает, что вы прочитали и согласны с пользовательским соглашением

http://www.mathnet.ru/rus/agreement

Параметры загрузки:

IP : 54.210 .77 .194

26 апреля 2023 г., $16: 16: 48$ 


\section{О ДЛИНЕ АЛГЕБРЫ ВЕРХНЕТРЕУГОЛЬНЫХ МАТРИЦ}

О. В. МАРКОВА

Задача вычисления длины полной матричной алгебры как функции порядка матриц была впервые поставлена в [1] и до сих пор не решена. В данной работе доказано, что длина алгебры верхнетреугольных матриц порядка $n$ и некоторых ее подалгебр специального вида равняется $n-1$, получены точные верхняя и нижняя оценки длины прямой суммы конечномерных алгебр и верхняя и нижняя оценки длины блочно-треугольных матричных подалгебр.

Пусть даны конечномерная алгебра $A$ над произвольным полем $F$ и $S$ - конечная система порождающих этой алгебры. Дадим определение длины данной системы порождающих и длины всей алгебры (см., например, [2]):

ОПредЕлЕниЕ 1. Длиной конечной системы порождающих $S$ для конечномерной алгебры $A$ называется наименьшее неотрицательное целое число $k$ такое, что слова от элементов $S$ длины, не превосходящей $k$, порождают $A$ как векторное пространство. Далее будем обозначать ее $l(S)$.

ОПредЕлЕниЕ 2. Длиной алгебры $A$ называется $l(A)=\max _{S} l(S)$, где максимум берется по всем конечньм системам порождающих этой алгебры.

Пусть далее $T_{n}(F)$ обозначает алгебру верхнетреугольных матриц порядка $n$ над полем $F$, $E$ - единичную матрицу, $E_{i j}$ - матричную единицу.

Теорема 1. Пусть $F-$ произвольное поле. Тогда $l\left(T_{n}(F)\right)=n-1$.

ДокАЗАТЕЛЬСтво. Пусть $A_{k}=\left(a_{i j}^{k}\right) \in T_{n}(F), k=1, \ldots, n$. Тогда

$$
\prod_{i=1}^{n}\left(A_{i}-a_{i i}^{i} E\right)=0 .
$$

Следовательно, произведение $A_{1} A_{2} \cdots A_{n}$ можно представить в виде линейной комбинации произведений матриц $A_{j}$, число сомножителей в которых меньше $n$, т.е. $l\left(T_{n}(F)\right) \leqslant n-1$. Рассмотрим следующую систему порождающих для $T_{n}(F)$ :

$$
S=\left\{E_{n n}, E_{i i}, E_{i, i+1}, i=1, \ldots, n-1\right\} .
$$

Так как $E_{1 n}=E_{12} E_{23} \cdots E_{n-1, n}$ не может быть представлена как произведение меньшего, чем $n-1$, числа элементов $S$, то $l(S)=n-1$. Следовательно, $l\left(T_{n}(F)\right) \geqslant n-1$.

СледСтвиЕ 1. Пусть $J \subseteq\{1, \ldots, n\}$. Через $A(J)$ обозначим подалгебру $T_{n}(F)$, состоящую из матрич вида $\sum_{j \in J} a_{j j} E_{j j}+\sum_{i, k=1, i<k}^{n} a_{i k} E_{i k}$. Тогда длина $A(J)$ равна $n-1$.

ДоКАЗАТЕЛЬСтво. Заметим, что если $J=\{1, \ldots, n\}$, то $A(J)$ совпадает с $T_{n}(F)$, и по доказанному выше $l(A(J))=n-1$. Пусть $J \subset\{1, \ldots, n\}$ и $A_{k}=\left(a_{i j}^{k}\right) \in A(J), k=1, \ldots, n$. Очевидно, что единичная матрица не является элементом $A(J)$, поэтому в данном случае требуется доказать, что произведение $A_{1} A_{2} \cdots A_{n}$ можно представить в виде линейной комбинации произведений длины от 1 до $n$. Это следует из равенства (1) и того, что $A_{j}-a_{j j}^{j} E=A_{j}$ для $j \notin J$. Значит, $l(A(J)) \leqslant n-1$. Система порождающих, на которой эта оценка достигается, такова: $S=\left\{E_{i, i+1}, i=1, \ldots, n-1\right\} \cup\left\{E_{j j}, j \in J\right\}$.

Перейдем к рассмотрению блочно-треугольного случая. Пусть $A$ и $B$ - конечномерные алгебры над одним полем $F$. Через $A \oplus B$ обозначим алгебру, элементами которой являются пары $(a, b), a \in A, b \in B$, с покоординатными сложением, умножением на числа и умножением.

Теорема 2. Пусть $A$ и $B$ - конечномерные алгебры над полем $F$ с длинами $l(A)$ и $l(B)$ соответственно. Тогда выполнено следующее неравенство:

$$
\max \{l(A), l(B)\} \leqslant l(A \oplus B) \leqslant l(A)+l(B)+1 .
$$


ДоКАЗАТЕльство. Обозначим $p=l(A), q=l(B)$. Для того чтобы доказать нижнюю оценку, возьмем системы порождающих $\left\{a_{1}, \ldots, a_{k}\right\}$ и $\left\{b_{1}, \ldots, b_{m}\right\}$ для $A$ и $B$ с длинами $p$ и $q$ соответственно. Тогда множество $\left\{\left(a_{1}, 0\right), \ldots,\left(a_{k}, 0\right),\left(0, b_{1}\right), \ldots,\left(0, b_{m}\right)\right\}$ будет системой порождающих в $A \oplus B$ длины $\max \{p, q\}$.

Возьмем в $A \oplus B$ произвольную систему порождающих $C=\left\{\left(c_{1}, d_{1}\right), \ldots,\left(c_{n}, d_{n}\right)\right\}$ и докажем, что всякое слово от элементов $C$ длины $p+q+2$ сократимо. Обозначим $N=p+q+2$. Пусть $v=\left(c_{i_{1}}, d_{i_{1}}\right) \ldots\left(c_{i_{N}}, d_{i_{N}}\right)=\left(c_{i_{1}} \ldots c_{i_{N}}, d_{i_{1}} \ldots d_{i_{N}}\right), i_{j} \in\{1, \ldots, n\}, j=1, \ldots, N$. Так как длина алгебры $A$ равна $p$, то слово $c_{i_{1}} \ldots c_{i_{p+1}}$ сократимо, т.е. $c_{i_{1}} \ldots c_{i_{p+1}}=\alpha_{1} c_{i_{1}} \ldots c_{i_{p}}+$ $\cdots+\alpha_{M-1} c_{n}+\alpha_{M} 1_{A}$. Так как длина алгебры $B$ равна $q$, то слово $d_{i_{p+2}} \ldots d_{i_{N}}$ сократимо, т.е. $d_{i_{p+2}} \ldots d_{i_{N}}=\beta_{1} d_{i_{p+2}} \ldots d_{i_{N-1}}+\cdots+\beta_{K-1} d_{n}+\beta_{K} 1_{B}$. Подставляя выражения для $c_{i_{1}} \ldots c_{i_{p+1}}$ и $d_{i_{p+2}} \ldots d_{i_{N}}$ в $v$, получим, что слово $v$ представляется в виде линейной комбинации слов меньшей длины. Так как $v$ было выбрано произвольно, то $l(A \oplus B) \leqslant p+q+1$.

Следующие примеры показьвают, что оценки (2) являются точными.

ПримеР 1 . Пусть $\mathbb{R}$ - поле действительных чисел, $A=T_{n}(\mathbb{R}), B=T_{m}(\mathbb{R})$. По теореме 1 $l(A)=n-1, l(B)=m-1$. Непосредственная проверка показьвает, что $l(A \oplus B)=n+m-1=$ $(n-1)+(m-1)+1=l(A)+l(B)+1$, что доказывает точность верхней оценки в $(2)$.

ПримеР 2. Пусть $N T_{n}(F)$ обозначает алгебру верхних нильтреугольных матриц порядка $n$ надполем $F$. Пусть $A=N T_{n}(F), B=N T_{m}(F)$. Из следствия 1 имеем $l(A)=n-1, l(B)=m-1$. Непосредственная проверка показьвает, что $l(A \oplus B)=\max \{n-1, m-1\}=\max \{l(A), l(B)\}$. Следовательно, нижняя оценка в (2) точна.

СлЕДСтвиЕ 2. Пусть $A_{1}, \ldots, A_{k}$ - конечномерные алгебры над полем $F$ с длинами $l_{1}, \ldots, l_{k}$ соответственно. Обозначим $A=A_{1} \oplus \cdots \oplus A_{k}$. Тогда выполнено следующее неравенство:

$$
\max \left\{l_{1}, \ldots, l_{k}\right\} \leqslant l(A) \leqslant \sum_{j=1}^{k} l_{j}+k-1 .
$$

Следующие примеры показьвают, что оценки (3) точны.

ПримеР 3. Пусть $D_{n}(F)$ - алгебра диагональных матриц порядка $n$ над полем $F$. Тогда если в поле $F$ найдется $n$ различных элементов, то по следствию из теоремы Гамильтона-Кэли $l\left(D_{n}(F)\right)=n-1$. Очевидно, что $D_{n}(F) \cong F \oplus \cdots \oplus F$, где в прямой сумме берется $n$ экземпляров $F$. Если $F=\mathbb{R}$ - поле действительных чисел, то $l\left(D_{n}(\mathbb{R})\right)=n-1=\sum_{j=1}^{n} l(\mathbb{R})+n-1$. Следовательно, верхняя оценка в (3) точна.

Пример 4. Возьмем $A_{i}=N T_{n_{i}}(F), i=1, \ldots, k$. Тогда, как и в примере 2 , получаем, что нижняя оценка в (3) точна.

СледСтвиЕ 3. Пусть $A$ - подалгебра блочно-диагональных или блочно-треугольных матрии, у которойна диагонали стоит $k$ блоков с длинами $l_{1}, \ldots, l_{k}$ соответственно. Тогда для $l(A)$ выполнено неравенство (3).

Автор выражает благодарность своему научному руководителю А. Э. Гутерману за постановку задачи и полезные обсуждения.

\section{СПИСОК ЛИТЕРАТУРЫ}

[1] A. Paz // Linear and Multilinear Algebra. 1984. V. 15. P. 161-170. [2] C. J. Pappacena // J. Algebra. 1997. V. 197. № 2. P. 535-545.

Московский государственньй университет им. М. В. Ломоносова
Представлено А. В. Михалевьм Принято редколлегией 22.07.2005 